\title{
METAZOÁRIOS PARASITOS DE SEIS ESPÉCIES DE PEIXES DO RESERVATÓRIO DE LAJES, ESTADO DO RIO DE JANEIRO, BRASIL*
}

\author{
ALINE R. PARAGUASSÚ; JOSÉ L. LUQUE ${ }^{2}$
}

\begin{abstract}
PARAGUASSÚ, A.R.; LUQUE, J.L. [Metazoan parasites of six fishes species from Lajes Reservoir in the State of Rio de Janeiro, Brazil.] Ecologia das comunidades de metazoários parasi-tos de seis espécies de peixes do Reservatório de Lajes, Estado do Rio de Janeiro, Brasil. Revista Brasileira de Parasitologia Veterinária, v. 16, n. 3, p. 121-128, 2007. Departamento de Parasitologia Animal, Instituto de Veterinária, Universidade Federal Rural do Rio de Janeiro, Caixa Postal 74508, Seropédica, RJ 23890-000, Brasil. E-mail: jlluque@ufrrj.br.

From April 2002 to July 2003, 231 freshwater fishes from Lajes Reservoir in the State of Rio de Janeiro, Brazil

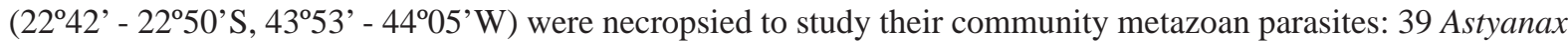
bimaculatus (Linnaeus, 1758), 79 A. fasciatus (Cuvier, 1819), 23 Hypostomus affinis (Steindachner, 1877), 26 Hoplias malabaricus (Bloch, 1794), 30 Loricariichthys castaneus (Castelnau, 1855) and 34 Trachelyopterus striatulus (Steindachner, 1876). The majority of specimens of $H$. affinis $(95.6 \%)$ and $H$. malabaricus (84.6\%) was parasitized by one or more metazoan species. In A. bimaculatus, A. fasciatus, L. castaneus and T. striatulus $41 \%$, $39.2 \%, 56.7$ and $14.7 \%$ of specimens were parasitized, respectively. Eight different metazoan parasites species were collected: 2 in A. bimaculatus, 3 in A. fasciatus, 3 in H. affinis, 4 in H. malabaricus, 4 in L. castaneus and 1 in $T$. striatulus. The parasites of the six host species showed the typical aggregated pattern of distribution. Two cases of negative correlation between host's total length and prevalence and parasite abundance were detected. The parasite community of $L$. castaneus showed the higher values of mean intensity, index of dispersion and higher values of diversity. The parasite communities of the studied fishes showed scarcity of significant correlations of parasitic abundance, species richness and diversity with the size of the host. The low species richness and diversity of parasite communities could be originated by the oligotrophic characteristics of the Lajes Reservoir.
\end{abstract}

KEY WORDS: Parasite ecology, community structure, freshwater fishes, Lajes Reservoir, Brazil.

\section{RESUMO}

Entre abril de 2002 e julho de 2003, foram necropsiados 231 espécimes de peixes: 39 Astyanax bimaculatus (Linnaeus, 1758), 79 A. fasciatus (Cuvier, 1819), 23 Hypostomus affinis (Steindachner, 1877), 26 Hoplias malabaricus (Bloch, 1794), 30 Loricariichthys castaneus (Castelnau, 1855) e 34 Trachelyopterus striatulus (Steindachner, 1876) provenien-

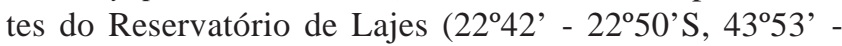
$\left.44^{\circ} 05^{\prime} \mathrm{O}\right)$, Estado do Rio de Janeiro, Brasil, para estudo das suas comunidades parasitárias. A maioria dos espécimes de

\footnotetext{
* Sob os aupícios do CNPq e Faperj.

${ }^{1}$ Curso de Pós-Graduação em Ciências Veterinárias, Universidade Federal Rural do Rio de Janeiro (UFRRJ), km 07 da BR 465, Seropédica, RJ 23890-000, Brasil. Bolsista Faperj.

${ }^{2}$ Departamento de Parasitologia Animal, Instituto de Veterinária, UFRRJ, Caixa Postal 74508, Seropédica, RJ 23851-970, Brasil. Bolsista do CNPq. E-mail: jlluque@ufrrj.br
}

H. affinis $(95,6 \%)$ e $H$. malabaricus $(84,6 \%)$ estavam parasitados por pelo menos uma espécie de metazoário. Em A. bimaculatus, A. fasciatus, $L$. castaneus e T. striatulus $41 \%$, $39,2 \%, 56,7 \%$ e $14,7 \%$ dos espécimes estavam parasitados, respectivamente. Foram coletadas oito diferentes espécies de metazoários parasitos: 2 em A. bimaculatus, 3 em A. fasciatus, $3 \mathrm{em} H$. affinis, $4 \mathrm{em} \mathrm{H}$. malabaricus, $4 \mathrm{em} L$. castaneus e 1 em T. striatulus. As comunidades de metazoários parasitos das seis espécies de peixes estudadas apresentaram típico padrão de distribuição agregada. Foram observados dois casos de correlação negativa entre a abundância e a prevalência parasitárias e o comprimento total dos hospedeiros. A comunidade parasitária de $L$. castaneus apresentou os maiores valores de intensidade média, índice de dispersão e diversidade. As comunidades parasitárias dos peixes estudados apresentaram escassez de correlação entre a abundância, riqueza parasitária e diversidade com o comprimento total dos hospedeiros. Os baixos valores de riqueza e diversidade das comuni- 
dades parasitárias podem ser atribuídos as características oligotróficas do Reservatório de Lajes.

PALAVRAS-CHAVE: Ecologia parasitária, estrutura da comunidade, peixes de água doce, Reservatório de Lajes, Brasil.

\section{INTRODUÇÃO}

O Reservatório de Lajes é o maior ambiente de águas represadas do Estado do Rio de Janeiro. Localiza-se nas verten-

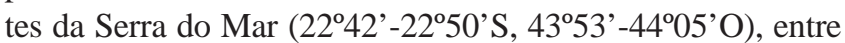
os municípios de Piraí e Rio Claro e a aproximadamente 80 km da cidade do Rio de Janeiro. Foi formado entre os anos de 1905 e 1908, através de águas desviadas do rio Piraí pelo túnel de Tocos e pelo represamento do Ribeirão das Lajes e de diversos outros tributários de pequeno porte como o rio da Prata e o rio do Pires (ARAÚJO et al., 1998). A represa ocupa cerca de 30 $\mathrm{km}^{2}$ de superfície ao nível de $415 \mathrm{~m}$ acima do mar, tendo como principal finalidade a geração de energia elétrica pela Light Serviços de Eletricidade S. A., atendendo parte da demanda do Estado (ARAÚJO; SANTOS, 2001). Suas águas são de elevada transparência ( $>2 \mathrm{~m}), \mathrm{pH}$ entre seis e sete e classificadas como oligotróficas, por apresentarem concentrações de nutrientes, nitrogênio e fósforo relativamente baixas, elevada transparência e baixas concentrações de pigmentos fotossintetizados por unidade de volume (DUARTE et al., 2002).

A ictiofauna da represa é composta por cerca de 26 espécies, dentre essas, espécies nativas como o acará, Geophagus brasiliensis (Quoy \& Gaimard, 1824); a traíra, Hoplias malabaricus (Bloch, 1794); o cascudo, Hypostomus affinis (Steindachner, 1877) e o cascudo-viola, Loricariichthys castaneus (Castelnau, 1855) e por espécies exóticas como o tucunaré, Cichla monoculus Agassiz, 1831 e a tilápia, Tilapia rendalli Boulenger, 1896, introduzidas em meados da década de 1950. Na década de 1990 ocorreu a introdução do pacuprata, Metynnis sp. e do tambaqui, Colossoma macropomum (Cuvier, 1818) para a pesca esportiva (SILVA et al., 1986; ARAÚJO; SANTOS, 2001).

No Brasil são escassos os estudos referentes às comunidades de metazoários parasitos de peixes de reservatórios. Até o presente momento, não foram realizados estudos que envolvessem uma análise parasitológica da ictiofauna, abrangendo várias espécies da mesma localidade. Os trabalhos existentes são de cunho taxonômico e/ou apresentam poucos dados quantitativos e, em sua maioria, são referentes a apenas uma espécie de metazoário parasito. A maioria desses estudos foi realizada com peixes provenientes do Reservatório de Volta Grande, Minas Gerais. Dentre esses podemos destacar os estudos realizados por: Martins et al. (1999, 2000a) que, respectivamente, registraram a ocorrência de Diplostomum sp. (Digenea: Diplostomatidae) e descreveram as larvas de Thynnascaris sp. (Nematoda: Anisakidae) na corvina de água doce Plagioscion squamosissimus (Heckel, 1840); por Martins et al. (2001a) com o registro de Ichthyouris voltagrandensis em pacu-manteiga (Myleus tiete) e Martins et al. (2000b, 2001b) a ocorrência do acantocéfalo Neoechinorhynchus curemai (Acanthocephala: Neoechinorhynchidae) em curimbatá, Prochilodus lineatus Valenciennes, 1836, apresentando dados quantitativos e histopatológicos dessa infecção. BrasilSato (2003) listou várias espécies de parasitos em peixes do reservatório de Três Marias (Rio São Francisco), em Minas Gerais. Feltran et al. (2004) realizaram um estudo de prevalência, abundância, intensidade e amplitude de infecção de nematóides intestinais em duas espécies de Leporinus, provenientes da represa de Nova Ponte, Perdizes, Minas Gerais. Recentemente, Moreira et al. (2005) realizaram uma análise dos aspectos ecológicos dos parasitos de Iheringichthys labrosus (Lütken, 1874) coletados de reservatórios da Bacia do Rio Paraná. Paraguassú et al. (2005) ao estudar a ecologia da comunidade de metazoários parasitos de Geophagus brasiliensis, realizaram o único estudo parasitológico de peixes do Reservatório de Lajes.

O presente trabalho tem como objetivo realizar o estudo de alguns aspectos ecológicos das comunidades de metazoários parasitos de Astyanax bimaculatus, A. fasciatus, $H$. affinis, H. malabaricus, L. castaneus e de Trachelyopterus striatulus, provenientes do Reservatório de Lajes, ao nível de comunidade componente e de infracomunidade, avaliando seus descritores quantitativos, e possíveis padrões de dominância e diversidade.

\section{MATERIAL E MÉTODOS}

Entre abril de 2002 e julho de 2003, foram necropsiados 231 hospedeiros: 39 espécimes de Astyanax bimaculatus, 79 de $A$. fasciatus, 23 de $H$. affinis, 26 de $H$. malabaricus, 30 de L. castaneus e 34 de T. striatulus provenientes do Reservatório de Lajes (22 $\left.42^{\circ}-22^{\circ} 50^{\prime} \mathrm{S}, 43^{\circ} 53^{\prime}-44^{\circ} 05^{\prime} \mathrm{O}\right)$, Estado do Rio de Janeiro. Foram realizadas 12 coletas de peixes: oito em 2002, de abril a setembro e quatro em 2003, de fevereiro a julho. Os peixes foram capturados com redes de espera $(50 \mathrm{~m}$ de comprimento por $3 \mathrm{~m}$ de altura), com malhas variando de 25 a 45 mm entre nós adjacentes e com tempo de permanência de 24 horas na considerada zona baixa da represa - situada nas proximidades da barragem, possuindo margens com relevos abruptos, maiores profundidades, e menores disponibilidades de estruturas submersas e abrigos. A identificação dos hospedeiros foi realizada de acordo com Bizerril e Primo (2001) e Reis et al. (2003).

Os espécimes estudados mediram: A. bimaculatus $11,9 \pm$ $1,8(8,0-16,0)$; A. fasciatus $13,8 \pm 1,2(11,0-17,7) ; H$. affinis $26,8 \pm 3,0$ (21,5 - 34,5); H. malabaricus 43,2 \pm 7,6 (16,0 58,7); L. castaneus $33,2 \pm 3,2(28,0-42,0)$ e $T$. striatulus $14,3 \pm 1,1(12,5-17,0) \mathrm{cm}$ de comprimento total.

Espécimes representativos das espécies de helmintos parasitos foram depositados na Coleção Helmintológica do Instituto Oswaldo Cruz (CHIOC), Rio de Janeiro, RJ. Astyanax bimaculatus: Rhabdochona acuminata (CHIOC 35436) e glossifonídeo não identificado (CHIOC 35437); A. fasciatus: R. acuminata (CHIOC 35438) e glossifonídeo não identifica- 
do (CHIOC 35439); H. affinis: dactilogirídeo não identificado (CHIOC 36615 a, b) e glossiphonídeo não identificado (CHIOC 35440); H. malabaricus: Ithyoclinostomum dimorphum (CHIOC 35433), Contracaecum sp. (CHIOC 35434) e glossiphonídeo não identificado (CHIOC 35435); L. castaneus: Austrodiplostomum sp. (CHIOC 36614 a, b) e glossiphonídeo não identificado (CHIOC 35441); T. striatulus: dactilogirídeo não identificado (CHIOC 36616).

A análise incluiu somente as espécies com prevalência maior que $10 \%$ (BUSH et al., 1990). O cálculo da freqüência de dominância e da dominância relativa (número de espécimes de uma espécie/número total de espécimes de todas as espécies de cada infracomunidade) foi feito seguindo a metodologia de Rohde et al. (1995). O quociente entre a variância e a abundância parasitária média (índice de dispersão, ID) foi calculado para cada espécie de parasito, com o intuito de determinar os padrões de distribuição, sendo sua significância testada com o estatístico $d$ (LUDWIG; REYNOLDS, 1988). Os dados referentes ao comprimento total dos hospedeiros, número total de parasitos, abundância, riqueza e diversidade parasitária foram transformados logaritmicamente $(\log x+1)$ para aproximação à distribuição normal (ZAR, 1996). Posteriormente, estes dados foram analisados pelo coeficiente de correlação de Pearson ( $r$ ) para verificar possíveis correlações com o comprimento total dos hospedeiros. O coeficiente de correlação de Pearson também foi utilizado para determinar a possível correlação entre o comprimento do hospedeiro e a prevalência da infecção/infestação parasitária, com prévia transformação angular dos dados de prevalência (ZAR, 1996). As amostras dos hospedeiros foram separadas da seguinte forma: $A$. bimaculatus em quatro intervalos com amplitude de $2 \mathrm{~cm}$, A. fasciatus em cinco intervalos de $1,5 \mathrm{~cm}, H$. affinis em cinco intervalos de 3 $\mathrm{cm}, H$. malabaricus em cinco intervalos de $8 \mathrm{~cm}, L$. castaneus em quatro intervalos de $5 \mathrm{~cm}$ e $T$. striatulus em cinco intervalos de $1 \mathrm{~cm}$.
O quociente entre a variância e a abundância parasitária média (índice de dispersão, ID) foi calculado para cada infracomunidade (LUDWIG; REYNOLDS, 1988). A diversidade parasitária foi calculada através do índice de Brillouin $(H)$, pois cada hospedeiro corresponde a uma comunidade mensurável em sua totalidade, utilizando para isso o logaritmo na base 10 (ZAR, 1996). A terminologia ecológica utilizada é a recomendada por Bush et al. (1997). O nível de significância estatística adotado foi $P<0,05$.

\section{RESULTADOS}

Comunidades componentes: Foram determinadas oito espécies diferentes de metazoários parasitos: dois digenéticos, um monogenético, dois nematóides, um isópode, um copépode e um hirudíneo (Tabela 1). Quatro de metazoários parasitos foram comuns a pelo menos duas comunidades. O glossiphonídeo não identificado (Hirudinea) foi encontrado em cinco das seis comunidades estudadas. Ergasilus sp. (Copepoda) foi registrado em quatro comunidades; $R$. acuminata (Molin, 1860) (Nematoda) e o dactilogirídeo não identificado (Monogenea) em duas comunidades (Tabela 1).

Astyanax bimaculatus: Foram coletadas duas espécies de metazoários parasitos: um nematóide e um hirudíneo. Rhabdochona acuminata foi à espécie mais prevalente e abundante (Tabela 1). O nematóide $R$. acuminata foi à espécie dominante com 86 espécimes, representando $97,7 \%$ do total de espécimes coletados, com o valor de dominância relativa média de $0,39 \pm 0,48$. O nematóide $R$. acuminata apresentou típico padrão de distribuição agregada (ID = 9,765; $d=18,58$ ).

Astyanax fasciatus: A comunidade parasitária está representada por três espécies de metazoários: um nematóide, um hirudíneo e um copépode. O nematóide $R$. acuminata foi a espécie mais prevalente e abundante (Tabela 1 ), representando $91,4 \%$ do total de espécimes coletados e com o valor de

Tabela 1. Prevalência (P\%) e abundância média ( $\mathrm{AM} \pm$ desvio padrão) dos metazoários parasitos de seis espécies de peixes do Reservatório de Lages, Estado do Rio de Janeiro, Brasil.

\begin{tabular}{|c|c|c|c|c|c|c|c|c|c|c|c|c|}
\hline \multirow[t]{2}{*}{ Parasitos } & \multicolumn{2}{|c|}{$\begin{array}{l}\text { Astyanax } \\
\text { bimaculatus }\end{array}$} & \multicolumn{2}{|c|}{$\begin{array}{c}A . \\
\text { fasciatus }\end{array}$} & \multicolumn{2}{|c|}{$\begin{array}{l}\text { Hypostomus } \\
\text { affinis }\end{array}$} & \multicolumn{2}{|c|}{$\begin{array}{c}\text { Hoplias } \\
\text { malabaricus }\end{array}$} & \multicolumn{2}{|c|}{$\begin{array}{c}\text { Loricariichthys } \\
\text { castaneus }\end{array}$} & \multicolumn{2}{|c|}{$\begin{array}{c}\text { Trachelyopterus } \\
\text { striatulus }\end{array}$} \\
\hline & $P(\%)$ & $\mathrm{AM}$ & $\mathrm{P}(\%)$ & $\mathrm{AM}$ & $\mathrm{P}(\%)$ & $\mathrm{AM}$ & $\mathrm{P}(\%)$ & AM & $\mathrm{P}(\%)$ & $\mathrm{AM}$ & $\mathrm{P}(\%)$ & $\mathrm{AM}$ \\
\hline \multicolumn{13}{|l|}{ Digenea } \\
\hline Austrodiplostomum sp. & - & - & - & - & - & - & - & - & 30 & $4,1 \pm 10,4$ & - & - \\
\hline Ithyoclinostomum dimorphum & $m-$ & - & - & - & - & - & 30,8 & $1,9 \pm 5,8$ & - & - & - & - \\
\hline \multicolumn{13}{|l|}{ Monogenea } \\
\hline Dactilogirídeo não ident. & - & - & - & - & 30,4 & $3,2 \pm 8,6$ & - & - & - & 14,7 & $0,3 \pm 0,8$ & - \\
\hline \multicolumn{13}{|l|}{ Nematoda } \\
\hline Contracaecum sp. & - & - & - & - & - & - & 57,7 & $5 \pm 1,7$ & & - & - & - \\
\hline Rhabdochona acuminata & 41 & $2,2 \pm 4,6$ & 36,7 & $1 \pm 2,3$ & - & - & - & - & & - & - & - \\
\hline \multicolumn{13}{|l|}{ Hirudinea } \\
\hline Glossiphonídeo não ident. & 5,1 & $0,1 \pm 0,2$ & 2,5 & $0,01 \pm 0,1$ & 91,3 & $4,8 \pm 5,4$ & 30,8 & $0,6 \pm 1,1$ & 26,7 & $0,7 \pm 1,3$ & - & - \\
\hline Isopoda & & & & & & & & & & & & \\
\hline Cimothoídeo não ident. & - & - & - & - & - & - & - & - & 3,3 & $0,1 \pm 0,5$ & - & - \\
\hline \multicolumn{13}{|l|}{ Copepoda } \\
\hline Ergasilus sp. & - & - & 3,8 & $0,1 \pm 0,7$ & 17,4 & $0,3 \pm 0,9$ & 7,7 & $0,07 \pm 0,2$ & 10 & $0,1 \pm 0,4$ & - & - \\
\hline
\end{tabular}


dominância relativa média de $0,36 \pm 0,48$. O nematóide $R$. acuminata apresentou típico padrão de distribuição agregada $(\mathrm{ID}=4,96 ; d=15,36)$.

Hypostomus affinis: Foram coletadas três espécies de metazoários parasitos: um monogenético, um hirudíneo e um copépode. O hirudíneo foi o parasito que apresentou o maior valor de prevalência e abundância média, representando 58\% do total de espécimes coletados (Tabela 1), com os maiores valores de dominância relativa média e freqüência de dominância $(0,77 \pm 0,37 ; 18)$. Os componentes da comunidade parasitária de $H$. affinis apresentaram típico padrão de distribuição agregada, sendo o monogenético dactilogirídeo o parasito que apresentou o maior índice de dispersão (ID = 23,45; $d=25,56$ ).

Hoplias malabaricus: Foram coletadas quatro espécies de metazoários parasitos: um digenético, um nematóide, um hirudíneo e um copépode. O digenético Ithyoclinostomum dimorphum representou 45,9\% do total de espécimes coletados. Contracaecum sp. (larva) foi a espécie mais prevalente e abundante (Tabela 1), apresentou os maiores valores de dominância relativa média e freqüência de dominância $(0,55 \pm 0,99 ; 10)$. Os componentes da comunidade parasitária de H. malabaricus apresentaram típico padrão de distribuição agregada, sendo $I$. dimorphum o parasito que apresentou o maior índice de dispersão (ID = 17,57; $d=22,64)$. O glossiphonídeo apresentou correlação negativa entre a abundância parasitária e o comprimento total do hospedeiro $(r=-0,529 ; P=0,005)$.

Loricariichthys castaneus: A comunidade parasitária está representada por quatro espécies de metazoários: um digenético, um hirudíneo, um isópode e um copépode. Austrodiplostomum sp. foi a espécie mais prevalente e abundante (Tabela 1). Este parasito representou $80,4 \%$ do total de espécimes coletados e com os maiores valores de dominância relativa média e freqüência de dominância $(0,29 \pm 0,45$; 9). Os componentes da comunidade parasitária de $L$. castaneus apresentaram típico padrão de distribuição agregada, sendo o digenético Austrodiplostomum sp. o parasito que apresentou o maior índice de dispersão (ID $=26,66 ; d=31,77$ ). $\mathrm{O}$ glossiphonídeo não identificado apresentou correlação negativa entre a prevalência parasitária e o comprimento total do hospedeiro $(r=-0,970 ; P=0,029)$.

Trachelyopterus striatulus: Foi coletada apenas uma espécie de metazoário parasito (Tabela 1). O monogenético apresentou típico padrão de distribuição agregada (ID = 11,79; $d=20,21)$ e não apresentou correlação entre o comprimento total do hospedeiro a abundância e a prevalência parasitárias.

Infracomunidades: Foram coletados 649 espécimes de parasitos. Destes, 193 (29,7\%) em H. affinis, 151 (23,2\%) em L. castaneus, 111 (17,1\%) em H. malabaricus, 96 (14,8\%) em A. fasciatus, 88 (13,6\%) em A. bimaculatus e $10(1,6 \%)$ em $T$. striatulus. As comunidades de metazoários parasitos apresentaram típico padrão de distribuição agregada, sendo o maior valor de dispersão observado em $L$. castaneus. Este hospedeiro também apresentou os maiores valores de riqueza e diversidade parasitárias (Tabela 2).

O número total de espécimes de ectoparasitos correspondeu a $100 \%$ em $H$. affinis e T. striatulus. As infracomunidades de endoparasitos adultos de $A$. bimaculatus e A. fasciatus corresponderam a $97,7 \%$ e $89,5 \%$, respectivamente, dos espécimes coletados nestas comunidades. As infracomunidades de endoparasitos larvais corresponderam a $84 \%$ e $81,4 \%$ dos espécimes coletados, respectivamente, da comunidade parasitária de H. malabaricus e L castaneus. Em A. fasciatus e L. castaneus o comprimento total do hospedeiro apresentou correlação positiva entre a riqueza e a abundância parasitárias $(r=0,278, P=0,013 ; r=0,380, P=0,038)$, respectivamente.

\section{DISCUSSÃO}

Os resultados obtidos no presente estudo indicam que os endoparasitos são os principais componentes das comunidades parasitárias de $A$. bimaculatus e $A$. fasciatus, onde se observou a dominância de nematóides. Em $H$. malabaricus e $L$. castaneus os estágios larvais de digenéticos foram dominantes. Nas comunidades parasitárias de H. affinis e T. striatulus ocorreu dominância dos ectoparasitos, onde se observou dominância numérica do hirudíneo e do monogenético, respectivamente.

A presença de larvas de parasitos em peixes dulcícolas tem sido registrada com freqüência (MACHADO et al., 1996; GUIDELLI et al., 2003). Na maioria dos casos as larvas de digenéticos encontrados em peixes de água doce são pertencentes às famílias Clinostomidae Lühe, 1901 e Diplostomidae Poirier, 1886 (FORTES; HOFFMANN, 1995; KOHN et al., 1995; EIRAS et al., 1999; ABDALLAH et al., 2004). Estes parasitos utilizam moluscos como primeiro hospedeiro intermediário, peixes como segundo hospedeiro intermediário e

Tabela 2. Prevalência, intensidade média, abundância média, índice de dispersão (ID), riqueza parasitária e o índice de Brillouin $(H)$ das infracomunidades parasitárias de seis espécies de peixes do Reservatório de Lajes, Estado do Rio de Janeiro, Brasil.

\begin{tabular}{|c|c|c|c|c|c|c|}
\hline Hospedeiros & Prevalência(\%) & Intensidademédia & Abundânciamédia & $\mathrm{ID}(d)$ & Riqueza parasitária & $H$ \\
\hline Astyanax bimaculatus & 41,0 & $5,50 \pm 6,12$ & $2,25 \pm 4,72$ & $9,91\left(18,78^{\star}\right)$ & $0,48 \pm 0,60$ & - \\
\hline A. fasciatus & 39,2 & $3,03 \pm 3,00$ & $1,18 \pm 2,38$ & $4,86\left(15^{\star}\right)$ & $0,45 \pm 0,59$ & - \\
\hline Hypostomus affinis & 95,6 & $8,77 \pm 9,57$ & $8,39 \pm 9,53$ & $10,82(-1,91)$ & $1,39 \pm 0,72$ & $0,24 \pm 0,21$ \\
\hline Hoplias malabaricus & 84,6 & $5,04 \pm 6,60$ & $4,27 \pm 6,33$ & $9,38\left(14,66^{\star}\right)$ & $1,27 \pm 0,83$ & $0,19 \pm 0,05$ \\
\hline Loricariichthys castaneus & 56,7 & $9 \pm 12,43$ & $5,10 \pm 10,29$ & $20,77\left(27,16^{\star}\right)$ & $0,73 \pm 0,69$ & $0,35 \pm 0,22$ \\
\hline Trachelyopterus striatulus & 14,7 & $2,00 \pm 1,41$ & $0,29 \pm 0,87$ & $14,7(-2,04)$ & $0,15 \pm 0,36$ & - \\
\hline
\end{tabular}

(d) teste estatístico $d ;\left(^{*}\right)$ valores significativos. 
as aves piscívoras como hospedeiros definitivos (DIAS et al., 2003a, b).

Em relação aos nematóides, observar-se que a maioria dos registros é realizada com larvas de Contracaecum sp. (Anisakidae) (KLOSS, 1966; FABIO, 1982; MACHADO et al., 1996; GUIDELLI et al., 2003; MARTINS et al., 2003; MADI; SILVA, 2005). Este parasito na fase adulta é encontrado, preferencialmente, em aves piscívoras (VICENTE et al., 1995). Os peixes podem atuar como hospedeiros intermediários ou como paratênicos sendo relatado o seu registro em várias espécies, o que vem demonstrando a ausência de especificidade quanto ao hospedeiro intermediário. Quando atuam como hospedeiro paratênico, os peixes adquirem o parasito pela predação de outros peixes menores, que por sua vez se infectam ingerindo copépodes, moluscos gastrópodos ou mesmo a própria larva de Contracaecum no seu estágio de vida livre (MADI; SILVA, 2005). Madi e Silva (2005) registraram larvas de Contracaecum sp. $\left(\mathrm{L}_{3}\right)$ em G. brasiliensis e ressaltaram que este peixe faz parte da dieta alimentar de $H$. malabaricus no reservatório de Jaguari, assim como observado por Santos et al. (2004) no Reservatório de Lajes. Este fato vem a corroborar com os resultados de Paraguassu et al. (2005) e deste trabalho, onde se observaram larvas de Contracaecum em G. brasiliensis e em H. malabaricus. Além disso, em G. brasiliensis Contracaecum sp. apresentou valores de prevalência e de abundância média de $14 \%$ e 0,2 \pm 0,4 (PARAGUASSU et al., 2005), respectivamente, enquanto em H. malabaricus apresentou valores de $57,7 \%$ e $5,00 \pm 1,77$, o que sugere que essa infecção seja cumulativa. Segundo Madi e Silva (2005), esse fato pode estar associado ao tamanho dos peixes piscívoros, como as traíras, mais difíceis de serem predados pelas aves, e com isso acumulariam maior quantidade de larvas de Contracaecum devido a sucessivas predações de peixes menores infectados.

As infrapopulações parasitárias nos peixes do presente estudo apresentaram típico padrão de distribuição agregada. Este fato foi observado nos estudos com as comunidades de peixes dulcícolas de diversas regiões do Brasil (MACHADO et al., 1996; ALVES et al., 2000; GUIDELLI et al., 2003; ABDALLAH et al., 2004; MOREIRA et al., 2005). Uma das características mais comuns em infecções de hospedeiros vertebrados por parasitos é a tendência destes apresentarem-se distribuídos de maneira agregada (ZUBEN, 1997). A distribuição agregada de parasitos dentro da população de hospedeiros está associada a fatores ambientais, antes de fatores de natureza demográfica. Entre esses fatores ambientais incluem-se mudanças nos parâmetros físicos do ambiente no tempo e no espaço, e principalmente diferenças na susceptibilidade do hospedeiro à infecção, que podem ser devido às diferenças imunológicas, comportamentais e aos dos fatores genéticos (ANDERSON; GORDON, 1982; PACALA; DOBSON, 1988). Estes dois últimos podem também influenciar a taxa de mortalidade dos parasitos e hospedeiros bem como criar heterogeneidade na dispersão de parasitos dentro da população de hospedeiros. Estes fatores ambientais fazem com que os parâmetros populacionais, que controlam o tamanho da população de parasitos, sejam variáveis aleatórias (ZUBEN, 1997).

No presente estudo observou-se que a prevalência e a abundância parasitária estão relacionadas com o índice de dispersão. Este fato foi constatado em A. bimaculatus, A. fasciatus e $L$. castaneus, onde as espécies com os maiores valores de prevalência e abundância parasitária foram as que apresentaram os maiores valores do índice de dispersão. Entretanto, em $H$. malabaricus e $H$. affinis ocorreu o inverso: os parasitos Contracaecum sp. e o glossophonídeo não identificado apresentaram os maiores valores de prevalência e abundância média, nos respectivos hospedeiros, porém com os menores valores para o índice de dispersão. Segundo Poulin (1993), a partir do momento em que a prevalência aumenta os parasitos passam a distribuir-se mais uniformemente entre os hospedeiros e a explorar uma crescente fração da população disponível desses hospedeiros. Desta forma, a proporção de hospedeiros não infectados decresce. Assim sendo, os níveis de agregação declinam com o aumento da prevalência e da abundância parasitárias. Se a prevalência aumentasse independente da abundância média a dispersão tenderia a ser uniforme, com o número de parasitos ocupando o máximo de hospedeiros. Entretanto, observa-se que a abundância não é uniforme devido principalmente à heterogeneidade de padrões de comportamento de aquisição dos parasitos.

A influência do comprimento do hospedeiro na composição qualitativa e quantitativa das infracomunidades parasitárias é um tópico bastante discutido. Entretanto, o que se observa nos estudos sobre comunidades parasitárias de peixes dulcícolas é uma enorme heterogeneidade de resultados (ALVES et al., 2000). No presente trabalho, apenas o hirudíneo glossiphonideo não identificado apresentou correlação negativa entre a abundância e a prevalência parasitárias e o comprimento de $H$. malabaricus e $L$. castaneus, respectivamente.

As comunidades parasitárias de $A$. bimaculatus e $A$. fasciatus, do presente estudo, apresentaram semelhança na sua composição, sendo a única diferença a presença do copépode Ergasilus sp. em A. fasciatus. Estes peixes possuem a mesma distribuição no Reservatório de Lajes, assim como apresentam grande semelhança na composição da dieta alimentar (ARAUJO; SANTOS, 2001). Ambos são considerados como omnívoros de superfície e fundo, alimentando-se de invertebrados aquáticos, algas, macrófitas e insetos (BIZZERIL; PRIMO, 2001; VILELLA et al., 2002). O principal componente das faunas parasitárias desses caracídeos, observado no presente trabalho, é o nematóide $R$. acuminata. Este parasito utiliza insetos como Ephemeroptera, Plecoptera e Trichoptera como hospedeiros intermediários (MORAVEC, 1972, 1998; HIRASAWA; URABE, 2003), itens alimentares comuns para Astyanax spp. (VILELLA et al., 2002). Kloss (1966) descreveu as espécies $R$. fasciata e $R$. australis parasitando A. fasciatus, A. schubarti e A. bimaculatus, respectivamente, provenientes do rio Mogi-Guaçu, São Paulo. Entretanto, Moravec (1972) considerou que ambas as espéci- 
es, acima citadas, são sinonímias de $R$. acuminata. Ainda sobre os estudo de Kloss (1966) podemos observar que o autor registrou maior número de helmintos parasitos de $A$. bimaculatus e $A$. fasciatus em relação ao presente estudo. Foram encontradas três espécies de digenéticos adultos, quatros nematóides, além de $R$. acuminata, e uma espécie de larva de nematóide. A maioria dos estudos com parasitos de Astyanax spp. foi realizado em ambiente lótico, o que justificaria a diferença na composição das faunas parasitárias devido às características bióticas e abióticas locais. É importante ressaltar que no presente estudo não foram encontrados digenéticos adultos nem nematóides camallanídeos em $A$. bimaculatus e A. fasciatus.

Os estudos sobre os parasitos de H. malabaricus mostram uma significante heterogeneidade na composição da fauna parasitária, marcada principalmente pelas diferenças geográficas. Entretanto, observa-se que a presença de determinados parasitos, como nematóides e digenéticos, está relacionada à posição na teia alimentar do que propriamente em relação à especificidade dos parasitos e ao ambiente, lótico ou lêntico. A traíra varia seu hábito alimentar ao longo de seu desenvolvimento ontogênico. Nas primeiras fases de vida, alimenta-se de plâncton, posteriormente, os insetos são os principais componentes da dieta alimentar passando na fase adulta a ser ictiófaga (BIZERRIL; PRIMO, 2001). Esta dieta variada sugere a possibilidade que a traíra, ao longo do seu desenvolvimento ontogênico, ingira uma gama de organismos que podem atuar como hospedeiros intermediários de diversos parasitos.

As infracomunidades parasitárias dos peixes estudados apresentaram baixa diversidade e riqueza parasitárias. Este resultado diverge dos estudos realizados por Machado et al. (1996) e Guidelli et al. (2003) com peixes provenientes do rio Paraná, e pode ser considerado como reflexo das características e/ou das condições ambientais. Porém, corrobora com o estudo realizado por Carney e Dick (2000), onde observou-se comunidades depauperadas e baixa riqueza parasitária em lagos oligotróficos. Segundo Kennedy (1993), o número de espécies de parasitos das infracomunidades é considerado reflexo do número de espécies de hospedeiros na mesma localidade, a capacidade de transmissão e infecção dos hospedeiros intermediários e definitivos. O Reservatório de Lajes possui ictiofauna com baixa diversidade de espécies, em relação a outros reservatórios mesotróficos (ARAUJO; SANTOS, 2001). Essa reduzida ictiofauna está associada, segundo os autores, às características desse ecossistema. O Reservatório de Lajes e seus afluentes constituem habitats isolados, pois desde a construção da barragem, em 1905, os peixes não têm como realizar a migração entre o ambiente lêntico (reservatório) e lôtico (rios tributários), além de não possuir um grande tributário que permita essa migração. Além disso, é considerado um ecossistema estabilizado, pois é de se esperar que, decorridos cerca de 50 anos de seu último alteamento, toda a matéria orgânica submersa tenha sido consumida ou incorporada nos ciclos químicos (BIZERRIL; PRIMO, 2001). Com relação aos aspectos abióticos, Lajes apresenta águas oligotróficas, com baixa produtividade, baixa entrada de nutrientes e oscilações marcantes do nível da água, que constituem fatores limitantes à disponibilidade de espaço e alimento às suas populações de peixes (SANTOS et al., 2004). As características físicas e químicas da água constituem fatores limitantes e determinantes na estabilização de comunidades de organismos aquáticos invertebrados, os quais podem atuar como hospedeiros intermediários e/ou paratênicos (SALGADO-MALDONADO; KENNEDY, 1997; MARCOGLIESE, 2001).

Agradecimentos:- Ao professor Francisco Gerson Araújo pelos espécimes de hospedeiros obtidos pelo convênio LIGHT/UFRRJ (Projeto PISCES).

\section{REFERÊNCIAS BIBLIOGRÁFICAS}

ABDALLAH, V.D.; AZEVEDO, R.K.; LUQUE, J.L. Metazoários parasitos dos lambaris Astyanax bimaculatus (Linnaeus, 1758), A.parahybae Eigenman, 1908 e Oligosarcus hepsetus (Cuvier, 1829) (Osteichthyes: Characidae), do Rio Guandu, Estado do Rio de Janeiro, Brasil. Revista Brasileira de Parasitologia Veterinária, v. 13, n. 2, p. 57-63, 2004.

ALVES, D.R.; LUQUE, J.L.; PARAGUASSÚ, A.R. Ectoparasitos da tilápia nilótica Oreochromis niloticus (Osteichthyes: Cichlidae) da estação de piscicultura da UFRRJ. Revista da Universidade Rural: Ciências da Vida, v. 22, n. 1, p. 81-85, 2000.

ANDERSON, R.M.; GORDON, D.M. Processes influencing the distribution of parasite numbers within host populations with special emphasis on parasite-induced host mortalities. Parasitology, v. 85, n. 2, p. 373-398, 1982.

ARAÚJO, F.G.; SANTOS, L.N. Distribution of fish assemblage in the Lajes'reservoir, Rio de Janeiro, Brasil. Revista Brasileira de Biologia, v. 61, n. 4, p. 563-576, 2001.

ARAÚJO, F. G.; FICHBERG, I.; DUARTE, S. Ciclo reprodutivo de Loricariichthys spixii (Steindachner, 1882) na Represa de Ribeirão das Lajes, RJ. Acta Biologica Leopoldensia, v. 20, n. 2, p. 309-318, 1998.

BIZERRIL, C.R.S.F.; PRIMO, P.B.S. Peixes de Águas interiores do Estado do Rio de Janeiro. Rio de Janeiro: FEEMAR-SEMADS, 2001. $417 \mathrm{p}$.

BRASIL-SATO, M. C. Parasitos de peixes da bacia do São Francisco. In: H.P. GODINHO; A.L. GODINHO (eds.). Águas, peixes e pescadores do São Francisco das Minas Gerais. Belo Horizonte: PUC Minas, 2003. p. 149-165.

BUSH, A.O.; AHO, J.M.; KENNEDY, C.R. Ecological versus phylogenetic determinants of helminth parasite community richness. Evolutionary Ecology, v. 4, n. 1, p. 1-20, 1990.

BUSH, A.O.; LAFFERTY, K.D.; LOTZ, J.M.; SHOSTAK, A.W. Parasitology meets ecology on its own terms: Margolis et al. revisited. Journal of Parasitology, v. 83, n. 4, p. 575583, 1997.

CARNEY, J.P.; DICK, T.A. Helminth communities of yellow 
perch (Perca flavescens (Mitchill)): determinants of pattern. Canadian Journal of Zoology, v. 78, p. 538-555, 2000.

DIAS, M.L.G.G.; EIRAS, J.C.; MACHADO, M.H.; SOUZA, G.T.R.; PAVANELLI, G.C. The life cycle of Clinostomum complanatum Rudolphi, 1814 (Digenea: Clinostomidae) on the floodplain of the high Paraná river, Brazil. Parasitology Research, v. 89, n. 6, p. 506-508, 2003.

DIAS, M.L.G.G.; SANTOS, M.J.; SOUZA, G.T.R.; MACHADO, M.H.; PAVANELLI, G.C. Scanning electron microscopy of Ithyoclinostomum dimorphum (Trematoda: Clinostomidae), a parasite of Ardea cocoi (Aves: Ardeidae). Parasitology Research, v. 90, n. 6, p. 335-358, 2003b.

DUARTE, S.; CAETANO, C.B.; VICENTINI R.N.; ARAÚJO, F.G. Distribuição e abundância relativa de cumbaca Trachelyopterus striatulus Steindachner (Osteichthyes, Auchenipteridae) no reservatório de Lajes, Rio de Janeiro, Brasil. Revista Brasileira de Zoologia, v. 19, n. 3, p. 925933, 2002.

EIRAS, J.C.; DIAS, M.L.; PAVANELLI, G.C.; MACHADO, M.H. MACHADO. Histological studies on the effects of Clinostomum marginatum (Digenea: Clinostomidae) in its second intermediate host Loricariichthys platymetopon (Osteichthyes, Loricariidae) of the upper Paraná, Brazil. Acta Scientiarum; v. 21, n. 2, p. 237-241, 1999.

FABIO, S.P. Sobre alguns nematoda parasitos de Hoplias malabaricus. Arquivos da Universidade Federal Rural do Rio de Janeiro, v. 5, n. 2, p. 179 - 186, 1982.

FELTRAN, R.B.; JÚNIOR, O.M.; PINESE, J. F.; TAKEMOTO, R.M. Prevalência, abundância, intensidade e amplitude de infecção de nematóides intestinais em Leporinus friderici (Bloch, 1794) e L. obtusidens (Valenciennes, 1836) (Pisces, Anostomidae), na represa de Nova Ponte (Perdizes, MG). Revista Brasileira de Zoociências, v.6, n.2, p. 169-179, 2004.

FORTES, E.; HOFFMAN, R.P. Levantamento da fauna parasitária de peixes do Lago Guaíba, Porto Alegre, Rio Grande do Sul, Brasil. Revista Brasileira de Medicina Veterinária, v. 17, n. 3, p. 107-111, 1995.

GUIDELLI, G.M., ISAAC, A., TAKEMOTO, R.M.; PAVANELLI, G.C. Endoparasite infracommunities of Hemisorubim platyrhynchos (Valenciennes, 1840) (Pisces: Pimelodidae) of the Baía River, upper Paraná river floodplain, Brazil: specific composition and ecological aspects. Brazilian Jornal of Biology, v. 63, n. 2, p. 261268, 2003.

HIRASAWA, R.; URABE, M. Ephemera strigata (Insecta: Ephemeroptera: Ephemeridae) is the intermediate host of the nematodes Rhabdochona denudata honshuensis and Rhabdochona coronacauda in Japan. Journal of Parasitology, v. 89, n. 3, p. 617-620, 2003.

KENNEDY, C.R. The dynamics of intestinal helminth communities in eels Anguilla anguilla in a smalll stream: long-term changes in richness and structure. Parasitology, v. 107, n. 1, p. 71-78, 1993.

KLOSS, G. R. Helmintos parasitos de espécies simpátricas de
Astyanax (Pisces: Characidae). Papéis Avulsos do Departamento de Zoologia de São Paulo, v. 18, n. 17, p. 189219, 1966.

KOHN, A.; FERNANDES, B.M.B.; BAPTISTA-FARIA, M.F.D. Metacercariae of Diplostomum (Austrodiplostomum) compactum (Trematoda, Diplostomidae) in the eyes of Plagioscion squamosissimus (Teleostei, Sciaenidae) from the Reservoir of the Hydroelectric Power Station of Itaipu, Brazil. Memórias do Instituto Oswaldo Cruz, v.90, n.3, p.341-344, 1995.

LUDWIG, J.A.; REYNOLDS, J.F. Statistical Ecology: A Primer on Methods and Computing. New York: WileyInterscience Publications, 1988. 337p.

MACHADO, M.H.; PAVANELLI, G.C.; TAKEMOTO, R.M. Structure and diversity of endoparasitic infracommunities and the trophic level of Psedoplatystoma corruscans and Schizodon borelli (Osteichthyes) of the high Paraná river. Memórias do Instituto Oswaldo Cruz, v. 91, n. 4, p. 441448, 1996.

MADI, R.R.; SILVA, M.S.R. Contracaecum Railliet \& Henry, 1912 (Nematoda, Anisakidae): o parasitismo relacionado à biologia de três espécies de peixes piscívoros no reservatório do Jaguari, SP. Revista Brasileira de Zoociências, v. 7, n. 1, p. 15-24, 2005.

MARCOGLIESE, D.J. Pursuing parasites upon the food chain: Implications of food web structure and function on parasite communities in aquatic systems. Acta Parasitologica, v. 46, n. 2, p. 82-93, 2001.

MARTINS, M. L.; YOSHITOSHI, E. R.; UMEKITA, H. Ichthyouris voltagrandensis n.sp (Nematoda: Pharyngodonidae) from Myleus tiete Eigenmann \& Norris, 1900 (Osteichthyes: Characidar) in the Volta Grande Reservoir, MG, Brazil. Revista Brasileira de Biologia, v. 61, n. 2, p. 305-310, 2001a.

MARTINS, M.L.; FUJIMOTO, R.Y.; NASCIMENTO, A.A.; MORAES, F.R. Ocorrência de Diplostomum sp. Nordmann, 1832 (Digenea: Diplostomatidae) em Plagioscion squamosissimus Heckel, 1840, proveniente do Reservatório de Volta Grande, MG, Brasil. Acta Scientiarum, v. 21, n. 2, p. 263-266, 1999.

MARTINS, M.L.; FUJIMOTO, R. Y.; MORAES, F.R.; ANDRADE, P.M.; NASCIMENTO, A.A.; MALHEIROS, E.B. Description and prevalence of Thynnascaris sp. larvae Dollfus, 1933 (Nematoda: Anisakidae) in Plagioscion squamosissimus Heckel, 1840 from Volta Grande Reservoir, state of Minas Gerais, Brazil. Revista Brasileira de Biologia, v. 60, n.3, p. 519-526, 2000a.

MARTINS, M. L.; FUJIMOTO, R.Y.; ANDRADE, P.M.; TAVARES-DIAS, M. Recent studies on Neoechinorhynchus curemai Noronha, 1973 (Acanthocephala: Neoechinorhynchidae) in Prochilodus lineatus Valenciennes, 1836, from Volta Grande Reservoir, MG, Brazil. Revista Brasileira de Biologia, v. 60, n.4, p. 673-682, 2000b.

MARTINS, M.L.; MORAES, F.R.; FUJIMOTO, R.Y.; 
ONAKA, E.M.; QUINTANA, C.I. F. Prevalence and histopathology of Neoechinorhynchus curemai Noronha, 1973 (Acanthocephala: Neoechinorhynchidae) in Prochilodus lineatus Valenciennes, 1836, from Volta Grande Reservoir, MG, Brazil. Revista Brasileira de Biologia, v. 61, n.3, p. 517-522, 2001b.

MARTINS, M.L.; SANTOS, R.S.; KAZUYUKI, T.; MARENGONI, N.G.; FUJIMOTO, R.Y. Infection and susceptibility of three fish species from the Paraná River, Presidente Epitácio, State of São Paulo, Brazil, to Contracaecum sp. Larvae (Nematoda: Anisakidae). Acta Scientiarum. Animal Sciences, v. 25, n. 1, p. 73-78, 2003.

MORAVEC, F. General characterization of the nematodes genus Rhabdochona with a revision of the South Americam species. Vestnik Ceskoslovenske Spolecnosti Zoologicke, v. 36, p. 29-46, 1972.

MORAVEC, F. Nematodes of freshwater fishes of the Neotropical Region. Praga: Academia, 1998. 464p.

MOREIRA, S.T.; ITO, K.F.; TAKEMOTO, R.M.; PAVANELLI, G.C. Ecological aspects of the parasites of Iheringichthys labrosus (Lütken, 1874) (Siluriformes: Pimelodidae) in reservoirs of Paraná basin and upper Paraná floodplain, Brazil. Acta Scientia Biologica, v. 27, n. 4, p. 317-322, 2005.

PACALA, S.W.; DOBSON, A.P. The relation between the number of parasites/host and host age: population dynamic causes and maximum likelihood estimation. Parasitology, v. 96, n. 1, p. 197-210, 1988.

PARAGUASSÚ, A.R.; LUQUE, J.L.; ALVES, D.R.. Metazoários parasitos do acará, Geophagus brasiliensis (Quoy \& Gaimard, 1824), (Osteichthyes: Cichlidae) do Reservatório de Lajes, Estado do Rio de Janeiro, Brasil. Revista Brasileira de Parasitologia Veterinária, v. 14, n. 1, p. 35-39. 2005.

POULIN, R. The disparity between observed and uniform distributions: a new look at parasite aggregation.
International Journal of Parasitology, v. 23, n. 7, p. 937944, 1993.

REIS, R.E.; KULLANDER, S.O.; FERRARIS, Jr.C.J. Check list of the freshwater fishes of South and Central America. Porto Alegre: EDIPUCRS, 2003. 742p.

ROHDE, K.; HAYWARD, C.; HEAP, M. Aspects of the ecology of metazoan ectoparasites of marine fishes. International Journal for Parasitology, v. 25, n. 8, p. 945970, 1995.

SALGADO-MALDONADO, G..; KENNEDY, C.R. Richness and similarity of helminth communities in the tropical cichlid fish Cichlasoma urophthalmus from the Yucatan Peninsula, Mexico. Parasitology, v. 114, p. 581-590, 1997.

SANTOS, A.F.G.N.; SANTOS, L.N.; ARAÚJO F.G. Water level influences on body conditions of Geophagus brasiliensis (Perciforme: Cichlidae) in a Brazilian oligotrophic reservoir. Neotropical Ichthyology, v. 2, n. 3, p. 151-156, 2004.

SILVA, S.L.O; MENDES, Z.C.; CRISÓSTOMO, L.C.; ARAÚJO, F.G. Resultados preliminares do levantamento ictiológico na represa de Ribeirão das Lajes, Rio de Janeiro. Publicações Avulsas do Museu Nacional, v. 65, p.87-90, 1986.

VILELLA, F.S.; BECKER, F.G.; HARTZ, S.M. Diet of Astyanax species (Teleostei, Characidae) in an Atlantic Forest River in Southern Brazil. Brazilian Archiver of Biology and Technology, v. 45, n. 2, p. 223-232, 2002.

VICENTE, J.J.; PINTO, R.M.; NORONHA, D.; GONÇALVES, L. Nematode parasites of Brazilian Ciconiiformes birds: a general survey with new records for species. Memórias do Instituto Oswaldo Cruz, v. 90, n. 3, p. 389-393, 1995.

ZAR, J.H. Biostatistical Analysis. 3 ed., Upper Saddle River: Prentice-Hall, 1996. 662 p.

ZUBEN, C.J.V. Implicações da agregação espacial de parasitas para dinâmica populacional na interação hospedeiroparasita. Revista de Saúde Pública, v. 31, n. 5, p. 523-530, 1997.

Recebido em 10 de abril de 2006.

Aceito para publicação em 30 de setembro de 2007. 